\title{
LINEAR TOPOLOGICAL INVARIANTS OF SPACES OF HOLOMORPHIC FUNCTIONS IN INFINITE DIMENSION
}

\author{
Nguyen Minh Ha and Le Mau Hai
}

Abstract

It is shown that if $E$ is a Frechet space with the strong dual $E^{*}$ then $H_{b}\left(E^{*}\right)$, the space of holomorphic functions on $E^{*}$ which are bounded on every bounded set in $E^{*}$, has the property $(D N)$ when $E \in(D N)$ and that $H_{b}\left(E^{*}\right) \in(\Omega)$ when $E \in(\Omega)$ and either $E^{*}$ has an absolute basis or $E$ is a Hilbert-Frechet-Montel space. Moreover the complementness of ideals $J(V)$ consisting of holomorphic functions on $E^{*}$ which are equal to 0 on $V$ in $H\left(E^{*}\right)$ for every nuclear Frechet space $E$ with $E \in(D N) \cap(\Omega)$ is stablished when $J(V)$ is finitely generated by continuous polynomials on $E^{*}$.

\section{Introduction}

Let $E$ be a Frechet space with a fundamental system of semi-norms $\left\{\|\cdot\|_{k}\right\}$. For each subset $B$ of $E$ we define a semi-norm $\|\cdot\|_{B}^{*}$ on $E^{*}$, the strongly dual space of $E$, with values in $[0,+\infty]$ by

$$
\|u\|_{B}^{*}=\sup \{|u(x)|: x \in B\} .
$$

We write

$$
\|\cdot\|_{k}^{*}=\|\cdot\|_{U_{k}}^{*} \text {, where } U_{k}=\left\{x \in E:\|x\|_{k} \leq 1\right\} .
$$

Using the notation we say that $E$ has the property

$$
\begin{aligned}
& \forall p \exists q \forall k \exists d, C>0:\|\cdot\|_{q}^{* 1+d} \leq C\|\cdot\|_{k}^{*}\|\cdot\|_{p}^{* d} \\
& \exists p \forall q \exists k, C>0:\|\cdot\|_{q}^{2} \leq C\|\cdot\|_{k}\|\cdot\|_{p} .
\end{aligned}
$$

Supported by the State Program for Fundamental Researches in Natural Sciences. 
The above properties and many others properties were introduced and investigated by Vogt (see [9-12]). In [9] Vogt has proved that $E \in(\Omega)$ if and only if for every $p \geq 1$ there exists $q$ such that for every $k$ we have $C, d>0$ with

$$
U_{q} \subseteq C r U_{p}+\frac{1}{r^{d}} U_{k}
$$

for all $r>0$.

In [3] Meise and Vogt have investigated the structure of spaces of holomorphic functions in the relation with the above linear topological invariants in nuclear spaces of infinite dimension.

The aim of the present paper is to continue the study of Meise and Vogt in the non-nuclear case.

In Section 1 we prove that $H_{b}\left(E^{*}\right)$, the space of holomorphic functions on $E^{*}$, which are bounded on every bounded set in $E^{*}$, has the property $(D N)$ if $E$ has also the property $(D N)$. We also prove that when $E^{*}$ has an absolute basis, $H\left(E^{*}\right) \in(\Omega)$ if $E \in(\Omega)$. Moreover we prove also that $H\left(E^{*}\right) \in(\Omega)$ for every Hilbert-Frechet-Montel space $E$ having the property $(\Omega)$.

Finally in Section 3 we consider completemented ideals of $H\left(E^{*}\right)$, the space of holomorphic functions on $E^{*}$ equipped with the compact-open topology when $E$ is a nuclear Frechet space. By applying the spliting Vogt's theorem we prove that if $V$ is an algebraic hypersurface in $E^{*}$ with $E \in(D N) \cap(\Omega)$, the space

$$
J(V)=\left\{f \in H\left(E^{*}\right):\left.f\right|_{V}=0\right\}
$$

is complemented in $H\left(E^{*}\right)$. Moreover a more general result is also established. That is if $V$ is an algebraic set of finite codimension in $E^{*}$ such that $J(V)$ is generated by finite number of continuous polynomials, $J(V)$ is complemented in $H\left(E^{*}\right)$ when $E \in(D N) \cap(\Omega)$.

\section{Properties $(D N)$ and $(\Omega)$}

In this section we prove the following theorem which was proved in $[\mathbf{3}]$ by Meise and Vogt for the nuclear case.

2.1. Theorem. Let $E$ be a Frechet space. Then

(i) $H_{b}\left(E^{*}\right) \in(D N)$ if $E \in(D N)$.

(ii) $H_{b}\left(E^{*}\right) \in(\Omega)$ if $E^{*}$ has an absolute basis.

Proof: (i) Assume that $E \in(D N)$. By Vogt [9], $E$ can be considered as a subspace of a space of the form $F \widehat{\otimes}_{\pi} s$, where $F$ is some Banach space 
and $s$ is the space of rapidly decreasing sequences. Since every bounded set in $E^{*}$ can be extended to a bounded set in $\left(F \widehat{\otimes}_{\pi} s\right)^{*} \cong F^{*} \widehat{\otimes}_{\pi} s^{*}$, it follows that $H_{b}\left(E^{*}\right)$ can be considered as a subspace of $H_{b}\left(F^{*} \widehat{\otimes}_{\pi} s^{*}\right)$. Since $H_{b}\left(F^{*} \times s^{*}\right) \cong H_{b}\left(F^{*}, H\left(s^{*}\right)\right)$ and $H_{b}\left(s^{*}\right) \in(D N)[\mathbf{3}]$, (i) is an immediate consequence of the following two assertions.

Assertion 1. $H_{b}\left(F^{*} \widehat{\otimes}_{\pi} s^{*}\right)$ is isomorphic to a subspace of $H_{b}\left(F^{*} \times s^{*}\right)$.

Assertion 2. $H_{b}\left(F^{*}, H\left(s^{*}\right)\right) \in(D N)$.

Proof of Assertion 1: We check that the form $f \rightarrow g=\left.f\right|_{F^{*} \times s^{*}}$ defines an isomorphic map from $H_{b}\left(F^{*} \widehat{\otimes}_{\pi} s^{*}\right)$ to a subspace of $H_{b}\left(F^{*} \times s^{*}\right)$.

Given $B$ a bounded set in $F^{*} \widehat{\otimes}_{\pi} s^{*}$. Since $F^{*}$ is a Banach space and $s^{*}$ a $(D F N)$-space we can find a neighbourhood $U$ of $0 \in s$ and $r>0$ such that $B \subseteq \operatorname{conv}\left(D_{r} \times U^{0}\right)$ where $D_{r}=\left\{x \in F^{*}:\left\|x^{*}\right\|<r\right\}$. For each $f \in H_{b}\left(F^{*} \widehat{\otimes}_{\pi} s^{*}\right)$ consider its Taylor expansion at $0 \in F^{*} \widehat{\otimes}_{\pi} s^{*}: f(\omega)=$ $\sum_{n \geq 0} P_{n} f(\omega)$ where $P_{n} f(\omega)=\frac{1}{2 \pi i} \int_{|t|=r} \frac{f(t \omega)}{t^{n+1}} d t$. Let $\pi: F^{*} \times s^{*} \rightarrow F^{*} \widehat{\otimes}_{\pi} s^{*}$ be the canonical map. We have

$$
\begin{aligned}
&\|f\|_{B} \leq\|f\|_{\operatorname{conv}\left(D_{r} \otimes U^{0}\right)} \\
&= \sup \left\{|f(\omega)|: \omega \in \operatorname{conv}\left(D_{r} \otimes U^{0}\right)\right\} \\
& \leq \sup \left\{\sum_{n \geq 0}\left|P_{n} f(\omega)\right|: \omega \in \operatorname{conv}\left(D_{r} \otimes U^{0}\right)\right\} \\
&= \sup \left\{\sum_{n \geq 0}\left|P_{n} f\left(\sum_{j \geq 1} \lambda_{j}\left(z_{j} \otimes u_{j}\right)\right)\right|\right. \\
&\left.: z_{j} \in D_{r}, u_{j} \in U^{0}, j \geq 1, \sum_{j \geq 1}\left|\lambda_{j}\right| \leq 1\right\} \\
& \sup \left\{\sum_{n \geq 0} \sum_{j_{1}, j_{2}, \ldots, j_{n} \geq 1}\left|\lambda_{j_{1}}\right|\left|\lambda_{j_{2}}\right| \ldots\left|\lambda_{j_{n}} \widehat{P_{n} f}\left(z_{j_{1}} \otimes u_{j_{1}}, \ldots, z_{j_{n}} \otimes u_{j_{n}}\right)\right|\right. \\
& \leq \sum_{n \geq 0} \sup \left\{\mid \widehat{P_{n} f}\left(z_{j_{1}} \otimes u_{j_{1}}, \ldots, u_{j} \in U_{j_{n}}^{0} \otimes j \geq 1, \sum_{n \geq 1}\left|\lambda_{j}\right| \leq 1\right\}\right. \\
& \sup \left\{\sum_{\left.j_{n}\right)} \mid z_{j} \in D_{r}, u_{j} \in U^{0} \forall j \geq 1\right\} \\
&\left.\sum_{j_{1}, \ldots, j_{n} \geq 1}|\ldots| \lambda_{j_{n}}\left|: \sum_{j \geq 1}\right| \lambda_{j} \mid \leq 1\right\}
\end{aligned}
$$




$$
\begin{aligned}
\leq & \sum_{n \geq 0} \frac{n^{2 n}}{(n !)^{2}} \sup \left\{\left|P_{n} f(z \otimes u)\right|: z \in D_{r}, u \in U^{0}\right\} \\
& \sup \left\{\left(\sum_{j \geq 1}\left|\lambda_{j}\right|\right): \sum_{j \geq 1}\left|\lambda_{j}\right| \leq 1\right\} \\
= & \sum_{n \geq 0} \frac{n^{2 n}}{(n !)^{2}} \sup \left\{\left|\frac{1}{2 \pi i} \int_{|\lambda|=\rho} \frac{f(\lambda(z \otimes u))}{\lambda^{n+1}} d \lambda\right|: z \in D_{r}, u \in U^{0}\right\} \\
= & \sum_{n \geq 0} \frac{n^{2 n}}{(n !)^{2}} \sup \left\{\left|\frac{1}{2 \pi i} \int_{|\lambda|=\rho} \frac{g((\lambda z, u))}{\lambda^{n+1}} d \lambda\right|: z \in D_{r}, u \in U^{0}\right\} \\
\leq & \sum_{n \geq 0} \frac{n^{2 n}}{(n !)^{2} \rho^{n}} \sup \left\{|g((\lambda z, u))|:|\lambda|=\rho, z \in D_{r}, u \in U^{0}\right\} \\
= & \sum_{n \geq 0} \frac{n^{2 n}}{(n !)^{2} \rho^{n}} \sup \left\{|g((z, u))|: z \in D_{\rho r}, u \in U^{0}\right\} \\
= & \|g\|_{D_{\rho r} \times U^{0}} \sum_{n \geq 0} \frac{n^{2 n}}{(n !)^{2} \rho^{n}}<\infty
\end{aligned}
$$

for $\rho$ sufficiently large, where $\widehat{P_{n} f}$ denotes the continuous symetric $n$ linear map associated to $P_{n} f$. Hence the map $f \longmapsto g=\left.f\right|_{F^{*} \times s^{*}}$ is isomorphic from $H_{b}\left(F^{*} \widehat{\otimes}_{\pi} s^{*}\right)$ to a subspace of $H_{b}\left(F^{*} \times s^{*}\right)$. The assertion is proved.

Proof of Assertion 2: It is easy to see that the topology of $H_{b}\left(F^{*}, H\left(s^{*}\right)\right)$ can be defined by the system of seminorms given by

$$
\|f\|_{(r, p)}=\sup \left\{\left\|P_{n} f(x)\right\|_{p}:\|x\|<r\right\}
$$

where $\left\{\|\cdot\|_{p}\right\}$ is a fundamental sequence of seminorms on $H\left(s^{*}\right)$. Since $H\left(s^{*}\right) \in(D N)[\mathbf{3}]$ there exists $p_{1}$ such that

$$
\forall p \exists p_{2}, C>0:\|\cdot\|_{p}^{2} \leq C\|\cdot\|_{p_{2}}\|\cdot\|_{p_{1}}
$$

on $H\left(s^{*}\right)$.

Then we have

$$
\begin{aligned}
\|f\|_{(r, p)}^{2} & \leq \sup \left\{C\left\|P_{n} f(x)\right\|_{p_{2}}\left\|P_{n} f(x)\right\|_{p_{1}}:\|x\|<r\right\} \\
& =\sup \left\{C r^{2 n}\left\|P_{n} f(x)\right\|_{p_{2}}\left\|P_{n} f(x)\right\|_{p_{1}}:\|x\|<1\right\} \\
& \leq C\|f\|_{\left(r^{2}, p_{2}\right)}\|f\|_{\left(1, p_{1}\right)} .
\end{aligned}
$$


The assertion 2 is proved.

Now we continue the proof of (ii) in Theorem 2.1. Let $\left\{e_{j}^{*}\right\}$ be an absolute basis of $E^{*}$ and $\left\{e_{j}\right\} \in E^{* *}$ be coefficient functionals. For each $p$ put

$$
N_{p}=\left\{j \in N:\left\|e_{j}^{*}\right\|_{p}^{*}<\infty\right\}
$$

and

$$
E^{*}(p)=\left\{x^{*} \in E^{*}:\left\|x^{*}\right\|_{p}=\sum_{j \in N_{p}}\left|e_{j}\left(x^{*}\right)\right|\left\|e_{j}^{*}\right\|_{p}^{*}<\infty\right\}
$$

Obviously $N_{p} \subseteq N_{p+1}$ for every $p \geq 1$. Since $E$ is a Frechet space it implies that every bounded set in $E^{*}$ is contained and bounded in some $E^{*}(p)$. Indeed, given $K$ a bounded set in $E^{*}$. Take $p$ such that $K$ is contained and bounded in $E_{p}^{*}$, where by $E_{p}^{*}$ we denote the Banach space associated to $p$. Since for each bounded set $B$ in $E$, the seminorm

$$
\sum_{j \geq 1} \mid e_{j}\left(x^{*}\right)\left\|e_{j}^{*}\right\|_{B}^{*}
$$

is continuous on $E_{p}^{*}$, we have

$$
C(B)=\sup \left\{\sum_{j \geq 1}\left|e_{j}\left(x^{*}\right)\right|\left\|e_{j}^{*}\right\|_{B}^{*}: x^{*} \in K\right\}<\infty .
$$

Assume that for every $q \geq p$ there exists $x_{q}^{*} \in K$ and $x_{q, j} \in U_{q}$ such that

$$
\sum_{j \geq 1}\left|e_{j}\left(x_{q}^{*}\right)\right|\left|e_{j}^{*}\left(x_{q, j}\right)\right| \geq 2 q .
$$

Now for each $q$ we take $m_{q}$ such that

$$
\sum_{1 \leq j \leq m_{q}}\left|e_{j}\left(x_{q}^{*}\right)\right|\left|e_{j}^{*}\left(x_{q, j}\right)\right| \geq q
$$

Then we have $C(B)=\infty$, where $B=\left\{x_{q, j}: 1 \leq j \leq m_{q}\right\}$. This is impossible, because $B$ is bounded in $E$. By the hypothesis $E \in(\Omega)$ for every $p$ there exists $q$ such that for every $k$ there exist $d, C>0$ for which

$$
\|\cdot\|_{q}^{* 1+d} \leq C\|\cdot\|_{k}^{*}\|\cdot\|_{p}^{* d}
$$


Put $a_{j, p}=\left\|e_{j}^{*}\right\|_{p}^{*}$ we have

$$
a_{j, q}^{1+d} \leq C a_{j, k} a_{j, p}^{d} \text { for all } j \geq 1
$$

Let

$$
\begin{aligned}
M & =\left\{m=\left(m_{1}, \ldots, m_{n}, 0, \ldots\right)\right\} \text { and } \\
M_{p} & =\left\{m \in M: m_{j}=0 \text { for } j \notin N_{p}\right\} .
\end{aligned}
$$

Now for each $m \in M$ and each $f \in H_{b}\left(E^{*}\right)$ put

$$
b_{m}(f)=\frac{1}{(2 \pi i)^{n}} \int_{\left|t_{1}\right|=1} \ldots \int_{\left|t_{n}\right|=1} \frac{f\left(t_{1} e_{1}^{*}+\cdots+t_{n} e_{n}^{*}\right)}{t^{m+1}} d t_{1} \ldots d t_{n}
$$

where $t^{m+1}=t_{1}^{m_{1}+1} \ldots t_{n}^{m_{n}+1}$.

By Ryan [7] the series $\sum_{m \in M} b_{m}(f) z^{m}$ is absolutely convergent and uniformly on every bounded set in $E^{*}$ to $f$. Moreover, since the map

$$
\xi \longmapsto \sum_{j \in N_{p}} \frac{\xi_{j} e_{j}^{*}}{\left\|e_{j}^{*}\right\|_{p}}
$$

is an isomorphism from $l^{1}$ onto $E^{*}(p)$, by Ryan [7], the topology of $H_{b}\left(E^{*}\right)$ can be defined by the system of seminorms $\|\cdot\|_{(\alpha, p)}$ given by

$$
\|f\|_{(\alpha, p)}=\sup _{m \in M_{p}}\left\{\alpha^{m}\left|b_{m}(f)\right| m^{m} / a_{\cdot, p}^{m}|m|^{|m|}\right\}
$$

where $\alpha \in R^{+}=\{r \in \mathbf{R}: r>0\}$ and $|m|=m_{1}+m_{2}+\cdots+m_{n}$. We check that for every $(\alpha, p)$ we can find $(\beta, q)$ such that for every $(\gamma, k)$ there exist $d^{\prime}, C^{\prime}>0$ such that

$$
W_{(\beta, q)} \subseteq C^{\prime} r^{d^{\prime}} W(\gamma, k)+\frac{1}{r} W_{(\alpha, p)} \text { for all } r>0
$$

where $W_{(\alpha, p)}$ denotes the unit ball in $H_{b}\left(E^{*}\right)$ defined by the semi-norm $\|\cdot\|_{(\alpha, p)}$.

Given $(\alpha, p)$. Put $\beta=2 \alpha$. Take $q$ such that for every $k$ there exist $d$, $C>0$ for wich

$$
a_{j, q}^{1+d} \leq C a_{j, k} a_{j, p}^{d} \text { for all } j \geq 1 .
$$

Given $(\gamma, k)$. We check that $(*)$ holds for $C^{\prime}=1$ and

$$
d^{\prime}=d_{1}=d+\log _{2} \gamma C / \beta
$$


Obviously $(*)$ holds for every $0<r \leq 1$ and every $d^{\prime}, C^{\prime}>0$. It remains to check that $(*)$ holds for every $r>1$ and $d^{\prime}=d_{1}, C^{\prime}=1$. Let $f \in W_{(\beta, q)}$. Put

$$
\begin{aligned}
& M_{p}^{1}=\left\{m \in M_{p}:|m|>\log _{2} r\right\} \\
& M_{p}^{2}=\left\{m \in M_{p}:|m|>\log _{2} r \text { and } \frac{1}{a_{\cdot, p}^{m}} \leq \frac{1}{r a_{\cdot, q}^{m}}\right\} \\
& M_{p}^{3}=M_{p} \backslash\left(M_{p}^{1} \cup M_{p}^{2}\right) .
\end{aligned}
$$

We have

$$
\begin{aligned}
\sup & \left\{\alpha^{m}\left|b_{m}\right| m^{m} / a_{\cdot, p}^{m}|m|^{|m|}: m \in M_{p}^{1}\right\} \\
& \leq \sup \left\{(1 / 2)^{|m|} \beta^{m}\left|b_{m}\right| m^{m} / a_{\cdot, q}^{m}|m|^{|m|}: m \in M_{p}^{1}\right\} \\
& \leq(1 / 2)^{\log _{2} r} \sup \left\{\beta^{m}\left|b_{m}\right| m^{m} / a_{\cdot, q}^{m}|m|^{|m|}: m \in M_{q}\right\} \leq \frac{1}{r}
\end{aligned}
$$

and

$$
\begin{aligned}
\sup & \left\{\alpha^{m}\left|b_{m}\right| m^{m} / a_{\cdot, p}^{m}|m|^{|m|}: m \in M_{p}^{2}\right\} \\
& \leq(1 / r) \sup \left\{\beta^{m}\left|b_{m}\right| m^{m} / a_{\cdot, q}^{m}|m|^{|m|}: m \in M_{p}^{2}\right\} \\
& \leq(1 / r) \sup \left\{\beta^{m}\left|b_{m}\right| m^{m} / a_{\cdot, q}^{m}|m|^{|m|}: m \in M_{q}\right\} \leq \frac{1}{r} .
\end{aligned}
$$

Thus

$$
\sum_{m \in\left(M \backslash M_{p}\right) \cup\left(M_{p}^{1} \cup M_{p}^{2}\right)} b_{m}(f) z^{m} \in(1 / r) W_{(\alpha, p)} \text { for all } r \geq 1 \text {. }
$$

It remains to show that

$$
\sum_{m \in M_{p}^{3}} b_{m}(f) z^{m} \in r^{d_{1}} W_{(\gamma, k)} .
$$

First we observe that

$$
1 / a_{\cdot, k}^{m} \leq r^{d} C^{|m|} / a_{\cdot, q}^{m} \text { for all } m \in M_{p}^{3} .
$$

Indeed, in the converse case we have

$$
1 / a_{\cdot, k}^{m}>r^{d} C^{|m|} / a_{\cdot, q}^{m} \text { for some } m \in M_{p}^{3} .
$$

On the other hand, since $m \notin M_{p}^{2}$ we have

$$
1 / a_{\cdot, p}^{m}>1 / r a_{\cdot, q}^{m} .
$$


Hence

$$
a_{\cdot, q}^{(1+d) m}>C^{|m|} a_{\cdot, k}^{m} a_{\cdot, p}^{d m} .
$$

This inequality shows that for $1 \leq j \leq n$ we have

$$
\left(a_{j, q}^{(1+d)}\right)^{m_{j}}>C^{m_{j}}\left(a_{j, k} a_{j, p}^{d}\right)^{m_{j}} .
$$

This is impossible, because

$$
a_{j, q}^{1+d} \leq C a_{j, k} a_{j, p}^{d} .
$$

Thus we have

$$
\begin{aligned}
\sup & \left\{\gamma^{m}\left|b_{m}\right| m^{m} / a_{\cdot, k}^{m}|m|^{|m|}: m \in M_{p}^{3}\right\} \\
& \leq r^{d} \sup \left\{(\gamma C / \beta)^{|m|} \beta^{m}\left|b_{m}\right| m^{m} / a_{\cdot, q}^{m}|m|^{|m|}: m \in M_{p}^{3}\right\} \\
& \leq r^{d}(\gamma C / \beta)^{\log _{2} r} \sup \left\{\beta^{m}\left|b_{m}\right| m^{m} / a_{\cdot, q}^{m}|m|^{|m|}: m \in M_{p}^{3}\right\} \leq r^{d_{1}} .
\end{aligned}
$$

This means that

$$
\sum_{m \in M_{p}^{3}} b_{m}(f) z^{m} \in r^{d_{1}} W_{\gamma, k)}
$$

and, hence,

$$
f \in r^{d_{1}} W_{(\gamma, k)}+\frac{1}{r} W_{(\alpha, p)} .
$$

The theorem is proved.

2.2 Theorem. Let $E$ be a Hilbert-Frechet-Montel space with $E \in(\Omega)$. Then $H\left(E^{*}\right)$, the space of holomorphic functions on $E^{*}$ equipped with the compact-open topology, has the property $(\Omega)$.

Proof: Applying the spliting Vogt's theorem for exact sequences of Hilbert-Frechet spaces [12], it follows that $E$ is isomorphic to a quotient space of $l^{2} \widehat{\otimes}_{\pi} s$. This is possible, because for $E$ we have a canonical exact sequence of the form

$$
O \rightarrow E \rightarrow \prod_{j} E_{j} \rightarrow \prod_{j} E_{j} \rightarrow 0
$$

where $E_{j}$ are Hilbert spaces.

Since $E$ is a Frechet-Montel space every bounded set in $E$ is the image of a compact set in $l^{2} \widehat{\otimes}_{\pi} s$, we infer that $E^{*}$ is contained as a subspace of $\left(l^{2} \widehat{\otimes}_{\pi} s\right)^{*} \cong l^{2} \widehat{\otimes}_{\pi} s^{*}$. On the other hand, since $l^{2} \widehat{\otimes}_{\pi} s^{*}$ has a fundamental system of Hilbert semi-norms by Colombeau and Mujica [1], $H\left(E^{*}\right)$ is a quotient space of $H_{b}\left(l^{2} \widehat{\otimes}_{\pi} s^{*}\right)$. Thus it suffices to check that $H_{b}\left(l^{2} \widehat{\otimes}_{\pi} s^{*}\right) \in(\Omega)$. The following lemma will solve that. 
2.3. Lemma. $H_{b}\left(l^{2} \widehat{\otimes}_{\pi} s^{*}\right)$ is isomorphic to a quotient space of $H_{b}\left(l^{2}\right) \widehat{\otimes}_{\pi} H_{b}\left(s^{*}\right)$.

Proof: Given

$$
(f, g) \in H_{b}\left(l^{2}\right) \times H_{b}\left(s^{*}\right) .
$$

Consider the Taylor expansion of $f$ and $g$ at $0 \in l^{2}$ and $0 \in s^{*}$ respectively

$$
f(u)=\sum_{n \geq 0} P_{n} f(u) \text { for } u \in l^{2}
$$

and

$$
g(v)=\sum_{n \geq 0} P_{n} g(v) \text { for } v \in s^{*}
$$

For each $n \geq 0$ by

$\chi_{n}: \underbrace{\left(l^{2} \widehat{\otimes}_{\pi} s^{*}\right) \times \cdots \times\left(l^{2} \widehat{\otimes}_{\pi} s^{*}\right)}_{n} \rightarrow\left(l^{2} \widehat{\otimes}_{\pi} s^{*}\right) \widehat{\otimes}_{\pi}\left(l^{2} \widehat{\otimes}_{\pi} s^{*}\right) \widehat{\otimes}_{\pi} \cdots \widehat{\otimes}_{\pi}\left(l^{2} \widehat{\otimes}_{\pi} s^{*}\right)$

we denote the canonical $n$-linear map and by

$$
\begin{aligned}
\mu_{n}:\left(l^{2} \widehat{\otimes}_{\pi} s^{*}\right) \widehat{\otimes}_{\pi}\left(l^{2} \widehat{\otimes}_{\pi} s^{*}\right) \widehat{\otimes}_{\pi} \cdots \widehat{\otimes}_{\pi}\left(l^{2} \widehat{\otimes}_{\pi} s^{*}\right) & \\
& \rightarrow \underbrace{\left(l^{2} \widehat{\otimes}_{\pi} l^{2} \widehat{\otimes}_{\pi} \cdots \widehat{\otimes}_{\pi} l^{2} \widehat{\otimes}_{\pi} l^{2}\right)}_{n} \widehat{\otimes}_{\pi}\left(s^{*} \widehat{\otimes}_{\pi} \cdots \widehat{\otimes}_{\pi} s^{*}\right)
\end{aligned}
$$

we denote the canonical isomorphism. Putting

$$
\widehat{P_{n}}(f, g)=\left(\widehat{P_{n} f} \otimes \widehat{P_{n} g}\right) \mu_{n} \chi_{n}
$$

we obtain a continuous symetric $n$-linear form on $\left(l^{2} \widehat{\otimes}_{\pi} s^{*}\right) \times \cdots \times\left(l^{2} \widehat{\otimes}_{\pi} s^{*}\right)$. Let $P_{n} f \otimes P_{n} g$ denote the $n$-homogeneous polynomial on $l^{2} \widehat{\otimes}_{\pi} s^{*}$ induced by $\widehat{P_{n}}(f, g)$. We have

$$
\begin{aligned}
\left(P_{n} f\right) & \otimes\left(P_{n} g\right)\left(\sum_{j \geq 1} u_{j} \otimes v_{j}\right) \\
& =\widehat{P_{n}}(f, g)\left(\sum_{j \geq 1} u_{j} \otimes v_{j}, \ldots, \sum_{j \geq 1} u_{j} \otimes v_{j}\right) \\
& =\sum_{j_{1}, j_{2}, \ldots, j_{n} \geq 1} \widehat{P_{n}}(f, g)\left(\left(u_{j_{1}} \otimes v_{j_{1}}, \ldots, u_{j_{n}} \otimes v_{j_{n}}\right)\right. \\
& =\sum_{j_{1}, j_{2}, \ldots, j_{n} \geq 1} \widehat{P_{n} f}\left(u_{j_{1}}, \ldots, u_{j_{n}}\right) \widehat{P_{n} g}\left(v_{j_{1}}, \ldots, v_{j_{n}}\right)
\end{aligned}
$$


Hence for every $r>0$ and every neighbourhood $V$ of $0 \in s$ we have

$$
\begin{aligned}
& \left\|\sum_{n \geq 0} P_{n} f \otimes P_{n} g\right\|_{B} \leq \sum_{n \geq 0}\left\|P_{n} f \otimes P_{n} g\right\|_{B} \leq \sum_{n \geq 0}\left\|P_{n} f \otimes P_{n} g\right\|_{\operatorname{conv}(r D \times V)} \\
& =\sum_{n \geq 0} \sup \left\{\left|P_{n} f \otimes P_{n} g(\omega)\right|: \omega \in \operatorname{conv}\left(r D \times V^{0}\right)\right\} \\
& =\sum_{n \geq 0} \sup \left\{\left|P_{n} f \otimes P_{n} g\left(\sum_{j \geq 1} \lambda_{j} u_{j} \otimes v_{j}\right)\right|\right. \\
& \left.: u_{j} \in r D, v_{j} \in V^{o}, \sum_{j \geq 1}\left|\lambda_{j}\right| \leq 1\right\} \\
& \leq \sum_{n \geq 0} \sup \left\{\sum_{j_{1}, j_{2}, \ldots, j_{n} \geq 1}\left|\lambda_{j_{1}}\right| \ldots\left|\lambda_{j_{n}}\right| \mid\left(P_{n} f \otimes P_{n} g\right)\left(\left(u_{j_{1}} \otimes v_{j_{1}}, \ldots, u_{j_{n}} \otimes v_{j_{n}}\right) \mid\right.\right. \\
& \left.: u_{j} \in r D, v_{j} \in V^{0}, \sum_{j \geq 1}\left|\lambda_{j}\right| \leq 1\right\} \\
& =\sum_{n \geq 0} \sup \left\{\sum_{j_{1}, j_{2}, \ldots, j_{n} \geq 1}\left|\lambda_{j_{1}}\right| \ldots\left|\lambda_{j_{n}}\right|\left|\widehat{P_{n} f}\left(u_{j_{1}}, \ldots, u_{j_{n}}\right)\right| \widehat{P_{n} g}\left(v_{j_{1}}, \ldots, v_{j_{n}}\right) \mid\right. \\
& \left.: u_{j} \in r D, v_{j} \in V^{0}, \sum_{j \geq 1}\left|\lambda_{j}\right| \leq 1\right\} \\
& \leq \sum_{n \geq 0} \frac{n^{n}}{n !} \frac{\|f\|_{\rho r D}}{\rho^{n}} \frac{n^{n}}{n !} \frac{\|g\|_{\rho V^{0}}}{\rho^{n}}\left(\sum_{j_{1}, j_{2}, \ldots, j_{n} \geq 1}\left|\lambda_{j_{1}}\right| \ldots\left|\lambda_{j_{n}}\right|\right) \\
& =\|f\|_{\rho r D}\|g\|_{\rho V^{0}} \sum_{n \geq 0}\left(\frac{n^{n}}{n ! \rho^{n}}\right)^{2}\left(\sum_{j \geq 1}\left|\lambda_{j}\right|\right)^{n} \\
& \leq C_{\rho}\|f\|_{\rho r D}\|g\|_{\rho V^{0}}
\end{aligned}
$$

for $\rho$ sufficiently large. Thus the form

$$
\theta(f, g)=\sum_{n \geq 0} P_{n} f \otimes P_{n} g
$$

define a continuous bilinear map from $H_{b}\left(l^{2}\right) \times H_{b}\left(s^{*}\right)$ to $H_{b}\left(l^{2} \widehat{\otimes}_{\pi} s^{*}\right)$ which induces a continuous linear map

$$
\widehat{\theta}: H_{b}\left(l^{2}\right) \widehat{\otimes}_{\pi} H_{b}\left(s^{*}\right) \rightarrow H_{b}\left(l^{2} \widehat{\otimes}_{\pi} s^{*}\right)
$$


It remains to check that $\widehat{\theta}$ is surjective. Given $f \in H_{b}\left(l^{2} \widehat{\otimes}_{\pi} s^{*}\right)$. Let $\left\{e_{j}\right\}$ and $\left\{e_{j}^{*}\right\}$ be the canonical bases of $s$ and $s^{*}$ respectively. Formally we have, for every $\sum_{k \geq 1} u_{k} \otimes v_{k} \in l^{2} \widehat{\otimes}_{\pi} s^{*}$, the following equalities

$$
\begin{aligned}
f & \left(\sum_{k \geq 1} u_{k} \otimes v_{k}\right) \\
= & \sum_{n \geq 0} P_{n} f\left(\sum_{k \geq 1} u_{k} \otimes v_{k}\right) \\
= & \sum_{n \geq 0} P_{n} f\left(\sum_{k \geq 1}\left(u_{k} \otimes \sum_{j \geq 1} e_{j}\left(v_{k}\right) e_{j}^{*}\right)\right) \\
= & \sum_{n \geq 0} P_{n} f\left(\sum_{j \geq 1}\left(\sum_{k \geq 1} e_{j}\left(v_{k}\right) u_{k}\right) \otimes e_{j}^{*}\right) \\
= & \sum_{n \geq 0} \sum_{j_{1}, \ldots, j_{n} \geq 1} \widehat{P_{n} f}\left(\left(\sum_{k \geq 1} e_{j_{1}}\left(v_{k}\right) u_{k} \otimes e_{j_{1}}^{*}\right), \ldots,\left(\sum_{k \geq 1} e_{j_{n}}\left(v_{k}\right) u_{k} \otimes e_{j_{n}}^{*}\right)\right) \\
= & \sum_{n \geq 0} \sum_{j_{1}, \ldots, j_{n} \geq 1} \sum_{k_{1}, \ldots, k_{n} \geq 1} \sum_{P_{n} f}\left(u_{k_{1}} \otimes e_{j_{1}}^{*}, \ldots, u_{k_{n}} \otimes e_{j_{n}}^{*}\right) e_{j_{1}}\left(v_{k_{1}}\right) \ldots e_{j_{n}}\left(v_{k_{n}}\right) \\
= & \sum_{n \geq 0} \sum_{j_{1}, \ldots, j_{n} \geq 1} \sum_{k_{1}, \ldots, k_{n} \geq 1} f\left(\cdot \otimes e_{j_{1}}^{*}, \ldots, \cdot \otimes e_{j_{n}}^{*}\right)\left(u_{k_{1}}, \ldots, u_{k_{n}}\right) \\
= & \sum_{n \geq 0} \sum_{j_{1}, \ldots, j_{n} \geq 1}\left(\left[P_{n} f\left(\cdot \otimes e_{j_{1}}^{*}, \ldots, \otimes e_{j_{n}}^{*}\right) \otimes\left(e_{j_{1}}(\cdot) \ldots e_{j_{n}}(\cdot)\left(v_{k_{1}}, \ldots, v_{j_{n}}(\cdot)\right)\right]\left(\sum_{k \geq 1} u_{k} \otimes v_{k}\right)\right.\right.
\end{aligned}
$$

On the other hand if for $r>0$ and $V$ as above, choose a neighbourhood $\tilde{V}$ of $0 \in s$ such that $\sum\left\|e_{j}^{*}\right\|_{\tilde{V}}^{*}\left\|e_{j}\right\|_{V}<1 / 2$ we get the following estimations

$$
\begin{aligned}
& \sum_{n \geq 0} \sum_{j_{1}, \ldots, j_{n} \geq 1} \sup \left\{\sum_{k_{1}, \ldots, k_{n} \geq 1}\left|\lambda_{k_{1}}\right| \ldots\left|\lambda_{k_{n}}\right| \widehat{P_{n} f}\left(u_{k_{1}} \otimes e_{j_{1}}^{*}, \ldots, u_{k_{n}} \otimes e_{j_{n}}^{*}\right) \mid\right. \\
& \left.\times\left|e_{j_{1}}\left(v_{k_{1}}\right)\right| \ldots\left|e_{j_{n}}\left(v_{k_{n}}\right)\right|: u_{k} \in r D, v_{k} \in V^{0}, \sum_{k \geq 1}\left|\lambda_{k}\right| \leq 1\right\}
\end{aligned}
$$




$$
\begin{aligned}
& \leq \sum_{n \geq 0} \sum_{j_{1}, \ldots, j_{n} \geq 1} \sup \left\{\sum_{k_{1}, \ldots, k_{n} \geq 1}\left|\lambda_{k_{1}}\right| \ldots\left|\lambda_{k_{n}}\right|\right. \\
& \left|\widehat{P_{n} f}\left(u_{k_{1}} \otimes \frac{e_{j_{1}}^{*}}{\left\|e_{j_{1}}^{*}\right\|_{\bar{V}}^{*}}, \ldots, u_{k_{n}} \otimes \frac{e_{j_{n}}^{*}}{\left\|e_{j_{n}}^{*}\right\|_{\bar{V}}^{*}}\right)\right| \\
& \left.\left\|e_{j_{1}}^{*}\right\|_{\bar{V}}^{*} \ldots\left\|e_{j_{n}}^{*}\right\|_{\bar{V}}^{*}\left|e_{j_{1}}\left(v_{k_{1}}\right)\right| \ldots\left|e_{j_{n}}\left(v_{k_{n}}\right)\right|: u_{k} \in r D, v_{k} \in V^{0}, \sum_{k \geq 1}\left|\lambda_{k}\right| \leq 1\right\} \\
& \leq \sum_{n \geq 0} \sum_{j_{1}, \ldots, j_{n} \geq 1} \sup \left\{\sum_{k_{1}, \ldots, k_{n} \geq 1}\left|\lambda_{k_{1}}\right| \ldots\left|\lambda_{k_{n}}\right| \frac{n^{n}}{n ! \rho^{n}}\|f\|_{\rho r D \otimes V^{0}}\right. \\
& \left.\left\|e_{j_{1}}^{*}\right\|_{\bar{V}}^{*}\left\|e_{j_{1}}\right\|_{V} \ldots\left\|e_{j_{n}}^{*}\right\|_{\bar{V}}^{*}\left\|e_{j_{n}}\right\|_{V}: u_{k} \in r D, v_{k} \in V^{0}, \sum_{k \geq 1}\left|\lambda_{k}\right| \leq 1\right\} \\
& =\|f\|_{\rho r D \otimes V^{0}} \sum_{n \geq 0} \frac{n^{n}}{n ! \rho^{n}}\left(\sum_{j \geq 1}\left\|e_{j}^{*}\right\|_{\bar{V}}^{*}\left\|e_{j}\right\|_{V}\right)^{n}\left(\sum_{k \geq 1}\left|\lambda_{k}\right|\right)^{n} \\
& \leq\|f\|_{\rho r D \otimes V^{0}} \sum_{n \geq 0} \frac{n^{n}}{n ! 2^{n} \rho^{n}} \leq C_{\rho, V}\|f\|_{\rho r D \otimes V^{0}}
\end{aligned}
$$

for $\rho$ sufficiently large.

Now for each $n \geq 0$ we define $\chi_{n}(f) \in H_{b}\left(l^{2}\right) \widehat{\otimes}_{\pi} H_{b}\left(s^{*}\right) \cong\left(l^{2} \times s^{*}\right)$ given by

$$
\chi_{n}(f)(u, v)=\sum_{j_{1}, \ldots, j_{n} \geq 1} P_{n} f\left(u \otimes e_{j_{1}}^{*}, \ldots, u \otimes e_{j_{n}}^{*}\right) e_{j_{1}}(v) \ldots e_{j_{n}}(v) .
$$

It follows that

$$
\begin{aligned}
\hat{\theta}\left(\chi_{n}(f)\right)\left(u_{1} \otimes\right. & \left.v_{1}, \ldots, u_{n} \otimes v_{n}\right) \\
& =\sum_{j_{1}, \ldots, j_{n} \geq 1} P_{n} f\left(u_{1} \otimes e_{j_{1}}^{*}, \ldots, u_{n} \otimes e_{j_{n}}^{*}\right) e_{j_{1}}\left(v_{1}\right) \ldots e_{j_{n}}\left(v_{n}\right) \\
& =P_{n} f\left(u_{1} \otimes v_{1}, \ldots, u_{n} \otimes v_{n}\right) .
\end{aligned}
$$

Thus for $g=\sum_{n \geq 0} \chi_{n}(f) \in H_{b}\left(l^{2}\right) \widehat{\otimes}_{\pi} H_{b}\left(s^{*}\right)$ we get $\widehat{\theta} g=f$.

To complete the proof of the theorem 2.2 it suffices prove the following. 
2.4. Proposition. Let $B$ be a Banach space and $E$ be a FrechetMontel space such that $E^{*}$ has an absolute basis. Then $H_{b}\left(B \times E^{*}\right) \in(\Omega)$ if $E \in(\Omega)$.

Proof: Since $E$ is Frechet-Montel, we have $H_{b}\left(B \times E^{*}\right) \cong H\left(E^{*}, H_{b}(B)\right)$. By the hypothesis $E^{*}$ has an absolute basis $\left\{e_{j}^{*}\right\}$ as in Theorem 2.1 (ii) the topology of $H_{b}\left(E^{*}, H_{b}(B)\right)$ can be defined by the system of seminorms given by

$$
\|f\|_{(\alpha, \rho, r)}=\sup \left\{\alpha^{m} \rho^{n}\left\|P_{n} b_{m}(f)\right\| m^{m} / a_{\cdot, p}^{m}|m|^{|m|}\right\}
$$

where

$$
a_{j, p}=\left\|e_{j}^{*}\right\|_{p}^{*} \text { for } j, p \geq 1 \text {. }
$$

As in the proof of Theorem 2.1 (ii) for each $(\alpha, \rho, p)$ take $\beta=2 \alpha, \eta=2 \rho$, and $d_{1}=d+\log _{2} \gamma \theta C / \beta$ we have

$$
W_{(\beta, \eta, q)} \subseteq r^{d_{1}} W_{(\gamma, \theta, k)}+\frac{1}{r} W_{(\alpha, \rho, p)}
$$

for all $r>0$, where for each $p$ take $q$ such that for each $k$ there exist $C$, $d>0$ for which

$$
a_{j, q}^{1+d} \leq C a_{j, k} a_{j, p}^{d} \text { for all } j \geq 1
$$

and $(\gamma, \theta, k)$ is given.

Consequently $H\left(E^{*}, H_{b}(B)\right)$ and, hence, $H_{b}\left(B \times E^{*}\right)$ has the property $(\Omega)$.

The proposition is proved.

\section{Complemented ideals in $H_{b}\left(E^{*}\right)$}

Let $V$ be an analytic set in a locally convex space $E$ and let

$$
J_{b}(V)=\left\{f \in H_{b}(E):\left.f\right|_{V}=0\right\}
$$

It is known $[2]$ that $J_{b}(V)$ is a complemented subspace of $H_{b}(E)$ when $\operatorname{dim} E<\infty$ and $V$ is an algebraic set in $E$. In this section we shall prove the above result for algebraic hypersurfaces in the space $E^{*}$, where $E$ is a nuclear Frechet space with $E \in(D N) \cap(\Omega)$.

3.1. Theorem. Let $E$ be a nuclear Frechet space such that $E \in$ $(D N) \cap(\Omega)$ and let $V$ an algebraic hypersurface in $E^{*}$. Then $J(V)$ is a 
complemented subspace of $H\left(E^{*}\right)$, where $H\left(E^{*}\right)$ is the space of holomorphic functions on $E^{*}$ and

$$
J(V)=\left\{f \in H\left(E^{*}\right):\left.f\right|_{V}=0\right\} .
$$

Proof: Considering the exact sequence of nuclear Frechet spaces

$$
O \rightarrow J(V) \rightarrow H\left(E^{*}\right) \rightarrow H\left(E^{*}\right) / J(V) \rightarrow O
$$

by the spliting Vogt's theorem [9] it suffices to check that $J(V) \in(\Omega)$ and $H\left(E^{*}\right) / J(V) \in(D N)$.

3.2. Proposition. Let $E$ be a Frechet space with the property $(\Omega)$ and let $V$ be an algebraic hypersurface in $E^{*}$. Then $J_{b}(V) \in(\Omega)$ if one of the following two conditions holds

(i) $E^{*}$ has an absolute basis

(ii) $E$ is a Hilbert-Frechet-Montel space.

Proof: By Theorem 2.1 and 2.2, $H_{b}\left(E^{*}\right) \in(\Omega)$. Let $P_{1}, \ldots, P_{m}$ be irreducible polynomials on $E^{*}$ such that

$$
V=Z\left(P_{1}, \ldots, P_{m}\right)
$$

where by $Z\left(P_{1}, \ldots, P_{m}\right)$ we denote the zero-set of $P_{1}, \ldots, P_{m}$. Such polynomials exist by the factoriality of the ring $\mathbf{C}\left[E^{*}\right]$ of continuous polynomials on $E^{*}$ (this can be proved as in [6] for the case where $E$ is a Banach space). Take a decomposition of $E^{*}, E^{*}=F_{1} \oplus \mathbf{C} e_{1}$ such that

$$
P_{1}\left(x_{1}^{*}+z_{1} e_{1}\right)=\sum_{0 \leq j \leq p_{1}} a_{j}^{1}\left(x_{1}^{*}\right) z_{1}^{j} \in C\left[F_{1}\right]\left[z_{1}\right] .
$$

Let $D_{1} \in \mathbf{C}\left[F_{1}\right]$ be the discriminant of $P_{1}$. Since $P_{1}$ is irreducible so $D_{1} \neq 0$ and, hence,

$$
G_{1}=F_{1} \backslash Z\left(D_{1}\right)=\left\{x_{1}^{*} \in F_{1}: P_{1}\left(x_{1}^{*}\right) \text { has different } p_{1} \text { solutions }\right\}
$$

is dense in $F_{1}$.

As in $[\mathbf{6}]$ the map $\theta$ from $\mathbf{C}\left[E^{*}\right] \oplus \mathbf{C}\left[F_{1}\right]\left[z_{1}\right] p_{1}$ to $\mathbf{C}\left[E^{*}\right]$ given by

$$
(g, r) \rightarrow P g+r
$$

is an isomorphism, where

$$
\mathbf{C}\left[F_{1}\right]\left[z_{1}\right] p_{1}=\left\{r \in \mathbf{C}\left[F_{1}\right]\left[z_{1}\right]: \text { degree }\left(\text { for } z_{1}\right)<p_{1}\right\}
$$


Since $\mathbf{C}\left[E^{*}\right]$ and $\mathbf{C}\left[F_{1}\right]$ are dense in $H_{b}\left(E^{*}\right)$ and $H_{b}\left(F_{1}\right)$ respectively, the map $\theta$ is extended to an isomorphism $\widehat{\theta}$ from $H_{b}\left(E^{*}\right) \oplus H_{b}\left(F_{1}\right)\left[z_{1}\right] p_{1}$. Thus every $f \in H_{b}\left(E^{*}\right)$ is written uniquely in the form

$$
f=P g(f)+r(f)
$$

where $g(f) \in H_{b}\left(E^{*}\right)$ and $r(f) \in H_{b}\left(F_{1}\right)\left[z_{1}\right] p_{1}$. Now given $f \in J_{b}(V)$. Write $f$ in the form $(*)$. Then

$$
r(f)\left(x_{1}^{*}, \cdot\right)=0 \text { for } x_{1}^{*} \in G_{1} .
$$

Since $G_{1}$ is dense in $F_{1}$, we have $r(f)=0$ and, hence, $f=P_{1} g_{1}$ with $g_{1}=r(f)$.

It follows that $g_{1} \mid Z\left(P_{2}, \ldots, P_{m}\right)=0$, because $Z\left(P_{1}\right), \ldots, Z\left(P_{m}\right)$ are irreducible branchs of $V$. Applying the above argument to $g_{1}$ and $P_{2}$ we can write $g_{1}=P_{2} g_{2}$ with $\left.g_{2}\right|_{Z\left(P_{3}, \ldots, P_{m}\right)}=0$. Continuing this progress we get

$$
f=P_{1} \cdot P_{2} \ldots P_{m} g_{m} \text { for some } g_{m} \in H_{b}\left(E^{*}\right) .
$$

Consequently

$$
J_{b}(V)=P_{1} \ldots P_{m} H_{b}\left(E^{*}\right) \cong H_{b}\left(E^{*}\right) \in(\Omega) .
$$

3.3. Proposition. Let $E$ be a Frechet space with the property $(D N)$ and $V$ an algebraic set in $E^{*}$ with $\operatorname{codim} V<\infty$. Then

$$
H_{b}\left(E^{*}\right) / J_{b}(V) \in(D N) \text {. }
$$

Proof: Let $P_{1}, P_{2}, \ldots, P_{m}$ be polynomials such that $V=Z\left(P_{1}, \ldots, P_{m}\right)$ where by $Z\left(P_{1}, \ldots, P_{m}\right)$ we denote the common zero-set of $P_{1}, P_{2}, \ldots, P_{m}$.

To simplify our reasoning we consider only the case $m=2$. Take a decomposition of $E^{*}, E^{*}=F \oplus \mathbf{C} e_{1} \oplus \mathbf{C} e_{2}$ such that

$$
\begin{aligned}
& P=P_{1}=a_{p} z_{1}^{p}+\sum_{0 \leq j \leq p-1} a_{j} z_{1}^{j} \\
& Q=P_{2}=b_{q} z_{2}^{q}+\sum_{0 \leq k \leq q-1} b_{k} z_{2}^{k}
\end{aligned}
$$

where $a_{p}, b_{q} \in \mathbf{C} \backslash\{0\}$ and $a_{j}$ and $b_{k}$ are continuous polynomials on $F$ and

$$
\begin{aligned}
f & =P g+\sum_{0 \leq j \leq p-1} a_{j}(f) z_{1}^{j} \\
& =P g+\sum_{0 \leq j \leq p-1} z_{1}^{j}\left[Q h_{j}+\sum_{0 \leq k \leq q-1} b_{k}^{j} z_{2}^{k}\right] \\
& =P g+Q \sum_{0 \leq j \leq p-1} h_{j} z_{1}^{j}+\sum_{0 \leq j \leq p-1} \sum_{0 \leq k \leq q-1} b_{k}^{j} z_{1}^{j} z_{2}^{k}
\end{aligned}
$$


where $g \in \mathbf{C}\left[E^{*}\right], a_{j}, h_{j} \in \mathbf{C}\left(F \oplus \mathbf{C} e_{2}\right)$ and $b_{k}^{j} \in \mathbf{C}[F]$. Then as in Proposition 3.2 every $f \in H_{b}\left(E^{*}\right)$ is written uniquely in the form $(*)$ with $g \in H_{b}\left(E^{*}\right), a_{j}, h_{j} \in H_{b}\left(F \oplus \mathbf{C} e_{1}\right)$ and $b_{k}^{j} \in H_{b}(F)$. Let $\theta$ be the canonical map from $H_{b}(F)$ to $W=H_{b}\left(E^{*}\right) / J_{b}(V)$. Obviously, $\theta$ is injective and defines $W$ as a finitely generated $H_{b}(F)$-module. Hence there exists monic polynomials $R$ and $S \in H_{b}(F)[X]$ such that

$$
R\left(z_{1}\right)=S\left(z_{2}\right)=0 .
$$

This implies that $\left(B \times\left(\mathbf{C} e_{1} \oplus \mathbf{C} e_{2}\right)\right) \cap V$ is bounded for every bounded set in $F$. Then it is easy to see that $\theta$ is an embedding. Let us note that

$$
W \cong Z=H_{b}(F)\left[z_{1}, z_{2}\right] p, q / J_{b}(V) \cap H_{b}(F)\left[z_{1}, z_{2}\right] p, q .
$$

Thus $b_{k}^{j}$ induces for each $0 \leq j \leq p-1$ and $0 \leq k \leq q-1$ a continuous linear map on $Z$ and hence every $f \in Z$ can be written in the form

$$
f=\sum_{0 \leq r \leq s} C_{r}(f) g_{r}
$$

where $C_{r}$ are continuous linear for $0 \leq r \leq s$ and $\left\{g_{r}\right\}$ is some finite system in $Z$. This yields that we can find $u$ such that for every $f \in Z$ there exists a monic polynomial $Q \in H_{b}(F)[X]$

$$
Q=X^{u}+d_{u-1}(f) X^{u-1}+\cdots+d_{0}(f)
$$

where $Q(f)=0$ and $d_{j}$ are continuous polynomials on $Z$ with values in $H_{b}(F)$.

To prove that $Z \in(D N)$ by Vogt [11] it suffices to shown that every continuous linear map from $\Lambda_{1}(\alpha)$ to $Z$ is bounded on a neighbourhood of $0 \in \Lambda_{1}(\alpha)$ for every exponent sequence $\alpha=\left\{\alpha_{n}\right\}$ where

$$
\Lambda_{1}(\alpha)=\left\{\{\xi\} \in \mathbf{C}^{N}: \sum_{j \geq 1}\left|\xi_{j}\right| r^{\alpha_{j}}<\infty \text { for } 0<r<1\right\} .
$$

Given such a map $T$. Since $d_{j} T$ are continuous polynomials on $\Lambda_{1}(\alpha)$ and $H_{b}(F) \in(D N)$ (Theorem 2.1) again by Vogt [11] these polynomials are bounded on some neighbourhood of $U$ of $0 \in \Lambda_{1}(\alpha)$. Then from the relation

$$
(T f)^{u}+d_{u-1}(T f)^{u-1}+\cdots+d_{0}(T f)=0, \text { for } f \in U
$$

it follows that $T$ is bounded on $U$. The proposition is proved.

Since $I H_{b}\left(E^{*}\right) \in(\Omega)$ for every finitely generated ideal $I$ in $\mathbf{C}\left[E^{*}\right]$ such that $I H_{b}\left(E^{*}\right)$ is closed using Proposition 3.3 we have 
3.4. Theorem. Let $E$ be a nuclear Frechet space with $E \in(D N) \cap(\Omega)$ and let $V$ be an algebraic set in $E^{*}$ such that $J(V)$ is finitely generated by finite number of polynomials. Then $(J V)$ is complemented in $H_{b}\left(E^{*}\right)$.

Proof: Let $P_{1}, \ldots, P_{m} \in \mathbf{C}\left[E^{*}\right]$ generate $J(V)$ and let $I$ denote the ideal in $\mathbf{C}\left[E^{*}\right]$ generated by $P_{1}, \ldots, P_{m}$. We consider only $m=2$ because the other cases are similar. Given $f \in J(V)$. As in Proposition 3.3 we can write $f$ as follows

$$
f=P g+Q h+\sum_{0 \leq j \leq p-1} \sum_{0 \leq k \leq q-1} b_{k}^{j}(x) z_{1}^{j} z_{2}^{k}
$$

for some decomposition of $E^{*}, E^{*}=F \oplus \mathbf{C} e_{1} \oplus \mathbf{C} e_{2}$. Then $\left.r\right|_{V}:=$ $\left.\sum_{0 \leq j \leq p-1} \sum_{0 \leq k \leq q-1} b_{k}^{j}(x) z_{1}^{j} z_{2}^{k}\right|_{V}=0$. Considering the Taylor expansion of each $b_{k}^{j}$ at $0 \in F$ we have

$$
r=\sum_{n \geq 0} \sum_{0 \leq j \leq p-1} \sum_{0 \leq k \leq q-1} P_{n} b_{k}^{j}(x) z_{1}^{j} z_{2}^{k} .
$$

Let $\pi: V \rightarrow F$ be the restriction of the canonical projection from $E^{*}$ onto $F$ to $V$. As in Proposition 3.3, $\pi$ is a branched cover. By $S$ we denote the branched locus of $\pi$. Let $x \in F \backslash S$. Then we can find a neighbourhood $V$ of $x$ in $F \backslash S$ such that

$$
\pi^{-1}(V)=\sqcup_{s} U_{s} \text { and } \pi: U_{s} \cong V \times u_{s} \text { for every } s,
$$

where $\pi^{-1}(X)=\left\{u_{s}\right\}$.

Since

$$
\left.\sum_{n \geq 0} \sum_{0 \leq j \leq p-1} \sum_{0 \leq k \leq q-1} P_{n} b_{k}^{j}(x) z_{1}^{j} z_{2}^{k}\right|_{U_{s}}=0
$$

for every $s$ we infer that

$$
\left.\sum_{0 \leq j \leq p-1} \sum_{0 \leq k \leq q-1} P_{n} b_{k}^{j}(x) z_{1}^{j} z_{2}^{k}\right|_{\pi^{-1}(V)}=0 .
$$

This implies that this function is equal to 0 on $V$ because $V \backslash \pi^{-1}(S)$ is dense in $V$. Thus $r$ can be approximated by elements of $I$. Consequently $I H\left(E^{*}\right)=\mathrm{Cl}\left(I H\left(E^{*}\right)\right)=J(V)$ and the theorem is proved.

Acknowledgements. The authors would like to express their's deep gratitude to Prof. Nguyen Van Khue for helpful suggestion and advices that let to improvements in the presentation of this paper. At the same time we thank the referee for his(her) helpful remarks. 


\title{
References
}

1. J. F. Colombeau And M. C. Mujica, "Holomorphic and differentiable mappings of uniform type," Functional Analysis, Holomorphy and Approximation Theory, J. A. Barroso, ed., North-Holland Math. Stud. 71, 1982, pp. 179-200.

2. P. B. Dijakov And B. S. Mitiagin, The structure of polynomial ideals in the algebra of entire functions, Studia Math. 68 (1980), 85-104.

3. R. MeISE AND D. Vogt, Structure of spaces of holomorphic functions on infinite dimensional polydics, Studia Math. 75 (1983), 235-253.

4. R. MEISE AND D. Vogt, Holomorphic functions of uniformly bounded type on nuclear Frechet spaces, Studia Math. 83 (1986), 147-166.

5. A. Pietsh, "Nuclear Locally Convex Spaces," Ergeb. Math. Grenzgeb 66, Springer-Verlag, 1972.

6. J. P. Ramis, "Sous-ensembles Analytiques d'une variete Banachique complexe," Springer-Verlag, 1970.

7. R. A. Ryan, Holomorphic mappings on $l_{1}$, Trans. Amer. Math. Soc. 302(2) (1987), 797-811.

8. H. Shaefer, "Topological Vector Spaces," Springer-Verlag, 1971.

9. D. Vogt, On two classes of F-spaces, Arch. Math. 45 (1985), $255-266$.

10. D. Vogt, Charakterisierung der Potenzreihenraume von endlichem Typ und ihre Folgerungen, Manuscripta Math. 37 (1982), 269-301.

11. D. Vogt, Frechtraume zwischen denen jede stelige linear Abbidung beschrankt ist., J. Reine Angew. Math. 345 (1983), 182-200.

12. D. VoGT, Interpolation of nuclear operators and a spliting theorem for exact sequence of Frechet spaces, Preprint.

\author{
Department of Mathematics \\ Pedagogical Institute I Hanoi \\ Tu liem, Hanoi \\ VIETNAM
}

Primera versió rebuda el 26 d'Octubre de 1993, darrera versió rebuda el 28 de Juny de 1994 